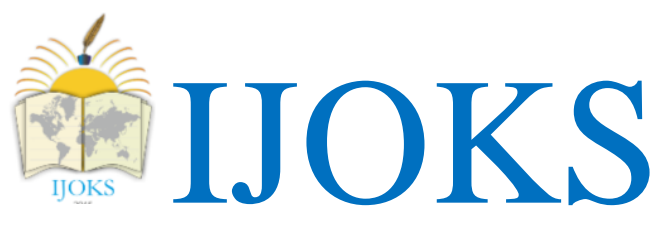

International Journal of Kurdish Studies

(ISSN:2149-2751)

$5(1)$, pp. $237-252$

http://www.ijoks.com

\title{
The structure of Verb phrase in the Kurdish language
}

\author{
Karwan Juma RAHEEM ${ }^{1}$
}

Received: Aug 07, 2018 Reviewed: Sep 21, 2018 Accepted: Sep 30, 2018

\begin{abstract}
Linguists define language as a means to communicate. There are many varieties of language in the world; each one is somewhat independent in some aspects, but generally shares some characteristics with all the others. Languages also tend to impact each other in various ways, especially by "borrowing" words through technology and politics. There is no doubt that every language has its own set of rules and characteristics such as grammar, sentence structure, and phonetics. This paper examines the structure of verb phrases in the Kurdish language the paper will clarify just what a verb phrase is. The X-Bar theory is used to illustrate the structure of verb phrasing in the Kurdish language. This theory dictates that each sentence structure has a "head," and all other sentence components are under the rule of this head. In sentences, the verb is the head and rules the sentence while in phrases, the head is usually either the noun or the verb. This research will illuminate the structure of verb phrasing in the Kurdish language and compare it with that of the English language.
\end{abstract}

Key words: Sentence structure, Verb phrase, linguistics, head, language, grammar, rule

\section{Recommended citation:}

Raheem, K.J. (2019). The structure of Verb phrase in the Kurdish language. International Journal of Kurdish Studies 5 (1), 237 - 252 DOI: 10.21600/ ijoks.516508

\section{Introduction}

Nowadays, there are many sciences that scientists are investigating the themes that they want and needed to investigate. So, related to languages a lot of linguists are working on a level of linguistics, which is syntax and it is a significant domain in linguistics and language field. The linguists mean to understand and figure out the compound systems of sentence structure and

\footnotetext{
${ }^{1}$ University of Charmo, College of Education and Natural Sciences, Department of Kurdish Language, Chamchamal, Sulaimani, Iraq. E-mail: Karwan.raheem@charmouniversity.org, ORCID : https://orcid.org/00000002-6735-0529
} 
they want to explain and simplify those systems for other people that they need to understand and study. Syntax illustrates that how the sentences are made and how the constituents of sentences come together in a certain language. It also demonstrates which type of constituents comes in the process of making the sentences. It is obvious that the sentences consist of the words but this process is not randomly, though the sentences make according complex systems in the syntax and grammar. The words come together for constructing the phrases and clauses then the sentences make of the phrases and clauses, which this system quite common between all languages. Therefore, English language applies this system for constructing its sentences and also Kurdish language cannot make its sentences without this system. This paper will investigate the structure of verb phrase in Kurdish language; it will indicate the rules of making verb phrase in that language. We will use a theory for describing and analysing the structure of verb phrase in Kurdish language, this theory proposed by Chomsky in 1980's in the governing and binding theory, that theory knows as X-bar theory. It works on many languages in the worldwide, which Kurdish language is one of them; in the analysing phrases by this theory Kurdish and English language are almost similar. Consequently, in this paper we will consider the structure of verb phrase in Kurdish language and compare it with English language in some examples. We will analyse the structure of verb phrase according the X-bar theory, which we will realise what are the constituents that participate in the constructing the verb phrases in Kurdish language.

The largest language family in the world is Indo-European language family, this family divided on some small groups for example Indo-Iranian language group. Kurdish language belongs to Indo-Iranian group in the Indo-European language family. In other words, this language is a branch of Indo-European language family but with Persian, Pashto, Baluchi, Dari are in a small group that called Indo-Iranian. Kurdish language mostly is using in western of Iran, Northern of Iraq and Syria and Eastern of Turkey. Moreover, in Northeast of Iran, Quchan, Neyshabur and North of Qazvin, which it was a great country called Kurdistan, Kurds dispersed after First World War between those countries. According to Blau (1989) Kurdish language includes in the Iranian languages in the North-western group. In general, Kurdish language divided on two main dialects, which first one is Kurmanji dialect and another one is Sorani dialect. In the parts of Northeast of Iran, Iraq, Syria, Caucasus and 
Anatolia Kurmanji dialect is used. Besides, Sorani dialect in the north of Iraq, west of Iran is spoken.

Regarding to the transcript and system of writing in Kurdish. So, there are some types of alphabets in the world that used by all of the languages such as Latin, Arabic, aria etc. Moreover, Kurdish language uses two kinds of writing system, which are Persia Arabic and Latin script. The Arabic form of transcript is mostly using with Sorani dialect. Another form of transcript is Latin, which is using with Kurmanji dialect. More, Kurdish language has 36 sounds or phonemes, which divided on 28 consonants and 8 vowels, also some diphthongs are using in this language (Zahedi \& Mehrazmay, 2011, p. 130- 134).

The main aim of grammar or syntax is defining the structure of sentences; which it is the largest grammar unit. So, sentence cannot define and explain without describe and determine the nature of those units or words that included in sentences. Syntax determines the type of the relations between them in the sentence frame. In every language, sentence consists of some basis; that each grammar unit can have a role as basis in the sentence, which the sentences comprise of phrases. The simplest sentence consists of two parts, in other words, a simple sentence contains two phrases, which are a noun phrase (NP) and a verb phrase (VP) (Amin, 2004, p. 199). Therefore, we understand that the smallest unit of grammar is sound and the biggest unit is sentence. In this paper we will work on syntax level and the structure of phrase more specific the structure of verb phrase in Kurdish language, which the phrases included in the syntax level of linguistics. It is most common among languages that sentence at least comprise of two categories, which are the subject category and the verb category. It will be clear in this example or rule:

$$
\begin{gathered}
\text { Sentence }=\text { noun phrase }+ \text { verb phrase } \\
\qquad S=N P+\text { VP }
\end{gathered}
$$

Concerning to the structure of phrase, now we will describe syntactic structure by focusing the strategy of putting the morphemes and words together for constructing the phrases and 
sentences. In other words, the process of making phrases and sentences will be illustrating by combined two or more constituents for making bigger constituent that occur in a process. This would be showed in the tree diagram that this occurs due to the rules of phrase structure. Here the rules of analysing the structure of phrases and sentences will be demonstrated. In each language sentence is the biggest syntactic unit and it based on few basics. Each syntactic unit can have a role as basic in the sentences construction; a simplest sentence consists of two phrases, which are noun phrase and verb phrase. The biggest syntactic unit known as sentence structure, hence, in the analysing the hierarchy of syntactic constituents in the tree diagram sentence is on the top and then analyses to middle constituents that phrase structure then until the last constituent, which is word structure. Each structure of sentence, phrase and word has its own rule of alignment the parts. Accordingly, the structure of phrase is in the middle of structure of sentence and structure of word. This means that it is smaller than sentence and bigger than word in term of structure. There are three structures in general that illustrated here:

Sentence structure (higher unit)

Phrase structure (middle unit)

Word structure (lower unit)

It clarified as tree diagram in this sentence for instance:

Azad hat.

Azad came.

Sentence

Noun phrase + verb phrase 


$$
\text { Noun + verb }
$$

There are two main basics in this sentence that are subject part and verb part, the noun phrase positioned in the subject part and the verb phrase positioned in the verb part. It means both subject and verb parts filled up by the noun phrase and verb phrase. Therefore, one or more of these syntactic categories (noun phrase, verb phrase, pronoun, adjective phrase, adverb phrase and prepositional phrase) can place in the sentence arguments. Each of phrase should has an independent word which is head, all of the words belong to the lexical categories that means they represent the lexical category of noun, pronoun, adjective, adverb, preposition and verb. Consequently, the phrases based on the basics of lexical categories, it means the head of each phrase should be the same as those lexical units. Since each phrase nominated and recognised according to its grammatical head for example, the head of noun phrase is noun and the head of verb phrase is verb that means the head is main in the phrases (Brzu, 2011, p. 12- 15).

Now, a question arise which is what is phrases, so, we need to answer this question and understand it in detail. Though, phrase has explicit connotation, in the linguistics field and in the daily speaking, phrases represent each collection of words. It will clarify in this two points, first point, in the formula of constituent, phrase ought to be a set of words. Second point, in the syntactic hierarchy, clauses are upper than phrases, automatically, it means clause are bigger than phrases in several senses. In other words, normally plain phrases do not comprise clauses; nevertheless typically basic clauses include phrases. Therefore, we reached an initial definition of phrase, which is a group of words refer to a phrase that inside a plain clause can task such a component (Kroeger, 2005, p. 35).

Phrase types

There are the types of the phrases

1- Noun phrase

2- Verb phrase

3- Adjective phrase an old man

have eaten

very happy (noun: man)

(verb: eaten)

(adjective: happy) 

4- Adverb phrase
very wisely
(adverb: wisely)
5- Prepositional phrase
in the school
(preposition: in)

Phrase as a technical word refer to even a single word, which is the main word solo. For instance, these two forms are adjective phrases, very happy and happy. The term phrase covers a minimum two or a series words in the normal use, which it appears to be abnormal that even a single word can be a phrase. Several rules that apply to adjective phrase, they can apply to adjective as well, which this a valid reason of the using the term broadly in syntax. This is an example that the rules apply to the places of very happy and happy in this sentence:

He was very happy.

He was happy.

It is straightforward to identify adjective phrase, which includes adjectives in place of stating adjective phrase or adjective every time (Greenbaum \& Nelson, 2002, p. 46- 47).

In general, the bases of the specific lexical and grammar classes based on the representation levels, under the name the syntactic levels and analysing levels. They recognised as phonetical form and logical form. In the lexicon, the classified items included in two kinds of categories, which are lexical categories and functional categories. The lexical categories include noun, verb, adjective and preposition, but another one is the functional categories, which consists of complement, inflectional, determiner, tense, agreement and negative. Related to the phrases, the number of the phrases changed from a language to another, in other words, the number and the productivity of the phrases different among languages, for example in Kurdish language adverb phrase exists but it is not active. So, the adjective phrase is not active as well, which sometimes it included in the verb phrase. Nevertheless, the noun phrase is very active in Kurdish language, or for adding those categories as addition recognised. All phrases must a have head; there is no any phrase without head, sometimes a phrase consists of one constituent that it becomes the head such as specific names or 
intransitive verb (Rasul, 2014, p. 32). Consequently, the verb phrase is active and productive than other types of the phrases except noun phrase in Kurdish language, therefore, we chose verb phrase to investigate in this paper, we will focus and write about it in detail from now.

In Kurdish language verb phrase is one of the main part or constituent in sentence, in a simplest form this main part consists of verb phrase. It should be looked as basis part and it is not optional. Hence, it has a great role in the constructing sentence. This type of phrase is more important than other types such as noun phrase and adjective phrase because it is mandatory part, while other type of phrases for example noun phrase may not emerge in the surface structure because of appear its sign inside the verb phrase. In other words, without verb phrase sentence never construct in the Kurdish language. Look at this for instance:

Azad hat.

Azad came.

Pro hat.

Pro came.

* Azad...... .

In the above structures, the first sentence comprise of a noun phrase and a verb phrase, in the next sentence the noun phrase did not emerge in the surface structure because of the subject agreement as person and number even it is zero morpheme inside the verb phrase. Nevertheless, in the last example only the noun phrase (Azad) expressed as subject thus this is ungrammatical sentence since in the structure of sentence verb phrase is a obligatory part and it cannot be omit (Brzu, 2011, p. 63-64). Therefore, we realised that there are two main parts for constructing sentence, which are noun and verb phrase parts. The noun phrase that its 
grammatical relation is subject can be omitted as pro subject in the surface structure and its sign emerge inside the verb phrase but the verb phrase cannot be omitted and it must come in the sentence.

Associated with the verb phrase, unlike the structure of noun phrase in Kurdish which is almost similar to English noun phrase, but the structure of verb phrase is different from English verb phrase. One of the most important constituents in the verbal category that its existence is significant and required is verb phrase. Within the verbal category verb expressed as head and one of its complements is noun phrase that its existence is required. Hence, a structure of verb phrase derives and it leaves an argument for the noun phrase and then it comes, which it formed here for example:

$$
\text { Verb }+ \text { Noun }=\text { verb phrase }
$$

It will be clarified in this example:

Pshilakak mndalakay trsand.

Cat the child the scared.

The cat scared the child.

Those verbs that participate in this rule are transitive verbs, in this example that unit that underlined is verb syntactic category, in this syntactic unit (trsand) is expressed as head and (mndalaka) is complement and it is required. Therefore, verb syntactic category is a structure of verb phrase. To know the role of the noun phrase in the structure of verb phrase an example clarify that how after omitting the noun phrase the sentence become an ungrammatical sentence:

*pshilaka trsand. 
Cat the scared.

The cat scared

*pshilaka mndalakay.

Cat the child the.

The cat the child.

The complement of the verb phrases that is a noun phrase or a phrase that has role as noun phrase because it can have placed in the noun phrase position. Therefore, the noun, adjective and adverb categories are not a part of the verb phrase lexically, this proved that it can be changed to other noun phrase but it should be matched with the meaning of the verb phrase. So, that noun phrase in the receiving the morpheme of subject person, it has the same behave as the other lexical categories, for instance:

Jutyaraka namameky jwani chand.

Farmer the tree a beautiful panted.

The farmer planted a beautiful tree.

Trem xward.

Grape I ate.

I ate grape.

(Maroof, 2010, p. 73- 74). 
Regarding to the structure of verb phrase, it has a complex structure, which means the structure of verb phrase has internal and external compound constituent. It can be expanded by various ways such as syntactic and morph syntactic extending, the root of the verb combine with those grammatical units that come in it which are morphemes and phrases. Each of them will make a type of grammatical relations; here, there are two types of structure of verb phrase, which are internal and external phrase structure.

\section{Internal structure of verb phrase}

The meaning of this is that according to the bond morphemes that play role in the constructing the constituent of the verb phrase structure. So, just by three morphemes the simplest verb phrase can be made that consists of the root of the verb, dependent pronoun morpheme with person and number and tense morpheme, look at these examples:

Root past tense morpheme dependent pronoun morpheme

$\mathrm{Ch}$

Chwm that means I went

$\mathrm{Br}$

d

W

$\mathrm{m}$

Brdm that means I took

$\mathrm{Kr}$

y

$\mathrm{m}$

Krym that means I bought

Here, the transitive and intransitive of present tense 
Aspect $\quad$ root + present morpheme dependent pronoun morpheme

$\mathrm{Da} \quad \mathrm{ch} \quad \mathrm{m}$

Dachm that means I am going

$\mathrm{Da} \quad \mathrm{ab} \quad \mathrm{m}$

Dabam that means I am taking

Verb consists of three bond morphemes, which are the basics of constructing verb phrase in those structures; the bond morphemes are root, tense and person. The root is the head of the verb; even this morpheme cannot come independently and it needs other morphemes to express the meaning, hence it carries the main meaning of the verb. The tense and person morphemes have a great role in the making the structure of verb phrase internally, in other words, these three morphemes are not optional they have to come in the constructing verb and verb phrase. Likewise, there is some other the grammatical and ungrammatical bond morphemes that participate in the constituent of verb, they attach to the root of the verb in the form of prefix and suffix. Look at these examples:

Nanaka nakhurawa.

The bread does not eat.

Nakhurawa

na, negative morpheme

kho, verb root

$\mathrm{r}$, invisible morpheme 
a, past tense morpheme

wa, near past tense morpheme

nasrin farshskay rakhstbu.

Nasrin carpet the opened up.

Nasrin opened up the carpet.

rakhstbu

ra, prefix

$\mathrm{kh}$, verb root

$\mathrm{t}$, past tense morpheme

bu, far past tense morpheme

(Brzu, 2011, p. 65- 67).

Therefore, the verb and its root cannot come alone in the structure as phrase in sentence, rather it has to receive and attach to some certain morphemes under the syntactic rules. Now, we understood the internal structure of verb and verb phrase in Kurdish language, and then we need to familiar the external structure of verb phrase that will be described in the next passages.

\section{The external structure of verb phrase}

In this, due to some grammatical units like morpheme, phrase and sentence verb phrase can be expanded, it means those grammatical units participate in the process of extending verb phrase. Some of those grammatical units function as additional and other as complements, this depend on the verb that has a great role in the choosing complements or extra information. 
Nevertheless, the incomplete verbs that are exist in Kurdish language cannot come alone, consequently, those verbs attach to all of the phrase types. In other words, the existence of those complement phrases is mandatory. The complements are:

1- Question marker

Ewa ke danasn?

You who know?

Who do you know?

2- Noun phrase

Mn wanakam xwendawa.

I lesson the read.

I read the lesson.

3- Pronoun phrase

Kchaka aw dabenet.

Girl the he seeing.

The girl is seeing him.

4- Prepositional phrase

Mndalaka kawta naw awakawa.

Child the fell down to water the.

The child fell down to the water

5- Noun phrase and prepositional phrase 
Awan Azad ba zirak dazann.

They Azad smart think.

They think that Azad is smart.

6- Pronoun phrase and prepositional phrase

Mn awanm ba pyaw zani.

I they men thought.

I thought they are men.

7- Adjective phrase

Nakhoshaka chak bu.

Sick the became good.

The sick became good.

8- Adverb phrase

Ali drang hat.

Ali late came.

Ali came late.

9- Quantity phrase

Mndalaka dwene shaw kam nust.

Mndal the last night a little slept.

The child slept a little last night. 
10- Complement sentence

Bistm, ka to ba yakam darchuit.

Heard I that you as first one graduated.

I heard that you graduated as first one.

Therefore, as we have mentioned above there are some verbs in Kurdish language that they choose what they need that is complement or no and also they choose what type of complement they require. Hence, without the complements the meaning of the verbs cannot be correct and the verbs have a great role in the choosing the appropriate complements to get appropriate meaning for the verb phrase and the sentences ( $\mathrm{Brzu}$, 2011, p. 68-70).

\section{Conclusion}

In conclusion, in today's modern world several sciences are exist and the researchers are doing research on the significant topics. Regarding to linguistic as science many linguists are investigating the languages and the property of languages across the world, one level of linguistic is more considered than others that is syntax, which has a great role to understand of a language thru this system. The researchers desire to point out the complex rules that they want to satisfy themselves and other people who work on languages. To understand the system of constructing the sentence, syntax has a great role. It shows the type of constituents that come in the making sentences and how they come and combine together. It is deniable that the sentences comprise the words but not arbitrarily, consequently, the sentences made up on the complex systems that syntax clarify them. For instance, the phrases made by the words then the cluses made by the phrases and the sentences made by the clauses. This type of rule is almost common among the languages, for example, making the English sentences based on this rule and certainly Kurdish language apply this rule. This paper has mentioned and described the structure of verb phrase in Kurdish language, the process of constructing the verb phrase have been explained and it analysed according the X-bar theory that it based on governing the other elements by the head. This theory has been described and it applied on Kurdish language. Due to the limitation of the time and number of the words, we could not describe the topic in detail any more but we have understood that what is the structure of the verb phrase and how it works in Kurdish language. 


\section{References}

Amin, W, O. (2004). Another light of linguistics, first volume, Aras book publisher, Hawler, Kurdistan Region, Iraq.

Brzu, O, B. (2011). The structure of phrase and Sentence in Kurdish language, Range printing press, Sulaimani, Kurdistan Region, Iraq.

Greenbaum, S \& Nelson, G. (2002). An Introduction to English Grammar, second edition, Longman, London.

Kroeger, P, R. (2005). Analysing Grammar, An Introduction, Cambridge University Press, New York.

Maroof, A, M. (2010). The structure of phrases in Kurdish language, Kurdology Centre, Sulaimani, Kurdistan Region, Iraq.

Rasul, S, H. (2014). Principles of economy in Kurdish language, published in Kurdish academic, Hawler, Kurdistan Region, Iraq.

Zahedi, K \& Mehrazmay, R. (2011). Definiteness in Sorani Kurdish and English, Dialectologia Revista electronica, 7 (2011), 129- 157. 